\title{
ON CERTAIN VARIETIES OF GROUPS WHICH ARE CENTRAL EXTENSIONS
}

\author{
R. A. BRYCE \\ (Received 13 April 1983) \\ Communicated by T. M. Gagen
}

\begin{abstract}
Connexions are sought between the subvarieties of a variety $\mathfrak{u}$ of groups and the subvarieties of the variety $[\mathfrak{U}, \mathfrak{E}]$ of all groups which are central extensions by groups in $\mathfrak{U}$, in the case when $\mathfrak{U}$ has the form $\mathfrak{A}, \mathfrak{B}$. Here $\mathfrak{A}_{r}$ is the variety of abelian groups of exponent dividing $r$ and $\mathfrak{B}$ is a variety of soluble groups of finite exponent.
\end{abstract}

1980 Mathematics subject classification (Amer. Math. Soc.): 20 E 10.

\section{Introduction}

Let $\mathfrak{U}$ be a variety of groups and, in the usual notation, let $[\mathfrak{U}, \mathfrak{E}]$ be the variety of all groups whose central factor groups are in $\mathfrak{U}$. An interesting problem is to determine relationships between the subvarieties of [ $\mathfrak{U}, \mathfrak{E}]$ and of $\mathfrak{U}$. In general significant results of this kind are difficult, if not impossible, to obtain, nilpotent varieties providing an obvious stumbling block. In [3] I tackled this question when $\mathfrak{U}$ is abelian-by-nilpotent and of finite exponent proving, roughly speaking, that the non-abelian-by-nilpotent subvarieties of [ $\mathfrak{U}, \mathbb{E}]$ are in natural one-to-one correspondence with non-nilpotent subvarieties of $\mathfrak{u}$. One could sum up that investigation, and the present one as examining aspects of non-nilpotence in certain varieties of the type $[\mathfrak{U}, \mathbb{E}]$.

In fact, the present paper is part of an attempt to investigate the method of [3] more closely and to use it on wider classes of varieties. The a priori constraints

(C) 1984 Australian Mathematical Society $0263-6115 / 84 \$ A 2.00+0.00$ 
that method imposes are several. First the subvarieties of [ $\mathfrak{U}$, ⿷匚] must be generated by finite groups (and therefore by critical groups: one relies on finding generating sets of groups with amenable structure and this is done, as usual, by analysing the structure of critical groups). This is ensured if $\mathfrak{u}$ is locally finite: see (1.3) below. Secondly, it is crucial that $\mathfrak{u}$ be contained in a variety of the form $\mathfrak{A} \mathfrak{B}$ with $\mathfrak{B}$ soluble, for one uses several times that abelian residuals of saturated formations are complemented in a finite soluble group (Huppert [5; VI, 7.15]). More importantly, perhaps, this set up gives rise to natural symplectic representations. The upshot is that we take $\mathfrak{U}$ to be $\mathfrak{A}, \mathfrak{B}$ where $r$ is greater than one and $\mathfrak{B}$ is a variety of soluble groups of finite exponent.

Now let $n$ be the least positive integer for which the groups in $\mathfrak{B}$ have nilpotent length at most $n$. We consider the varieties generated by the, so to say, "new" critical groups in $\left[\mathfrak{A}_{r} \mathfrak{B}, \mathbb{E}\right.$ ]. To be precise let $\mathscr{Q}$ be the set of subvarieties of $[\mathfrak{A}, \mathfrak{B}, \mathbb{E}$ ] which are generated by basic groups of nilpotent length greater than $n$ not in $\mathfrak{A}, \mathfrak{B}$. Similarly, let $\mathcal{E}$ be the set of subvarieties of $\mathfrak{A}_{r} \mathfrak{B}$ generated by basic groups of nilpotent length greater than $n$. We shall see (in (4.6)) that there exists a function $\theta: \mathcal{Q} \rightarrow \mathcal{L}$ defined by

$$
\mathfrak{X} \theta=\operatorname{var}\left\{G / \zeta_{1}(G): G \in \mathfrak{X}\right\} .
$$

The best result one can have is that $\theta$ is one-to-one, and one result ((4.6)) I prove is:

\section{(1.1) THEOREM. If either}

(a) $r$ is odd,

(b) $\mathfrak{B}$ is nilpotent, or

(c) the exponent of $\mathfrak{B}$ is odd,

then $\theta$ is one-to-one.

Of course the nilpotent case is (5.1) of [3], but all cases are instances of a common phenomenon as we shall see in Section 4. By contrast $\theta$ is not one-to-one in even the smallest cases excluded by the hypotheses of (1.1), namely, when $r=2$ and $\mathfrak{B}=\mathfrak{A}_{m} \mathfrak{A}_{2}$ with $m$ being odd (I give an explicit example to illustrate this in Section 4).

In a sequel I hope to examine contexts in which $\theta$ fails to be one-to-one. For example, for the varieties just mentioned:

(1.2) Theorem. Let $r=2$ and $\mathfrak{B}=\mathfrak{A}_{m} \mathfrak{A}_{2}$ with $m$ odd. Then, if $\mathfrak{L} \in \mathfrak{L}$ is join-irreducible, $\mathfrak{R} \theta^{-1}$ contains at least one and at most two join-irreducible varieties.

Unexplained notation and results concerning varieties of groups follow Hanna Neumann's book [9]. At several points I rely heavily on results and methods from [3]: this paper should be read in close conjunction with it. 
To conclude this introduction I prove the following result: in one sense it underlies the whole philosophy of this paper.

(1.3) LEMMA. Let $\mathfrak{u}$ be a locally finite variety of groups. Every subvariety of $[\mathfrak{U}, \mathbb{E}]$ is generated by finite groups.

Proof. Every variety is generated by finitely generated groups so it suffices to show that if $G \in[\mathfrak{U}, \mathbb{E}]$ is finitely generated than $\operatorname{var} G$ is generated by finite groups.

To this end note that $G / \zeta_{1}(G)$ is finite, being in $\mathfrak{U}$ and finitely generated. By Neumann [8] then, $G^{\prime}$ is finite, say of order $N$. Also $\zeta_{1}(G)$ is finitely generated since it has finite index in a finitely generated group. Let the torsion subgroup $T$ of $\zeta_{1}(G)$ have exponent $e$. Then $G^{\prime} \cap \zeta_{1}(G)^{e}=1$. For, if $x \in G^{\prime} \cap \zeta_{1}(G)^{e}$ then $x=z^{e}$ for some $z \in \zeta_{1}(G)$; so $1=x^{N}=z^{e N}$ and $z \in T$; hence $x=1$. Now $G$ is a subdirect product of $G / G^{\prime}$ and $G / \zeta_{1}(G)^{e}$, the first being abelian and the second finite. Hence $\operatorname{var} G$ is generated by finite groups.

\section{Quotations from [3]}

We shall need several results from [3] or, at any rate, results much like them. The reader will be referred there for proofs which are similar enough to warrant omitting here.

Let $\mathfrak{B}$ be a variety of soluble groups of finite exponent, say of nilpotent length $n$ with $n$ minimal. Let $G$ be a basic group in $[\mathfrak{A}, \mathfrak{B}, \mathbb{E}]$ which is of nilpotent length greater than $n$ and not in $\mathfrak{A}, \mathfrak{B}$. The notation of the next result will be carried through the whole paper, often without comment.

(2.1) LEMMA. Let $\S_{G}$ be the set of groups $S$ with the properties

(i) $\operatorname{var} S=\operatorname{var} G$,

(ii) the nth nilpotent residual of $S$ is complemented in $S$, and

(iii) $S$ is s-critical.

Then $\mathcal{S}_{G}$ is not empty and if $R$ is a group of least order in $\delta_{G}$ it has the following structure. Let $A$ be the nth nilpotent residual of $R$ and $B$ a complement for it in $R$.

(a) $A$ is a p-group, for some prime $p$ dividing $r$, of class 2;

(b) $\zeta_{1}(A)=A^{\prime}=\zeta_{1}(R)$ is cyclic;

(c) $O_{p^{\prime}}(B) \neq 1$;

(d) if $N$ is a non-trivial normal $p^{\prime}$-subgroup of $B$ then $[A, N]=A$;

(e) $\mathrm{C}_{R}\left(A / A^{\prime}\right)=A$;

(f) $A=\mathfrak{B}(R)$; and

(g) $R$ is a basic group. 
The proof of (2.1) is little different from that of (3.4) in [3] and its immediate preamble.

Let $\Re$ denote the set of minimal elements of all $S_{G}$ as $G$ ranges over the basic groups in $[\mathfrak{A}, \mathfrak{B}, \mathbb{E}]$ which have nilpotent length greater than $n$ and which are not in $\mathfrak{A}_{r} \mathfrak{B}$; much of the remainder of the paper is concerned with the structure of the groups in $\Re$. A variety is in the set $\mathscr{Q}$ introduced in the Introduction if and only if it is generated by a subset of $\Re$.

We recall now the central construction from [3]. Let $B$ be a group, $p^{\alpha}$ a prime-power and $M$ a $Z_{p^{a}} B$-module. The $B$-group $M^{\dagger}$ is defined on the set $M \times M^{*} \times Z_{p^{\alpha}}\left(M^{*}\right.$ is the contragredient of $\left.M\right)$ by the operation

$$
\left(m_{1}, f_{1}, z_{1}\right)\left(m_{2}, f_{2}, z_{2}\right)=\left(m_{1}+m_{2}, f_{1}+f_{2}, z_{1}+z_{2}-f_{1}\left(m_{2}\right)\right)
$$

where $m_{1}, m_{2} \in M, f_{1}, f_{2} \in M^{*}$ and $z_{1}, z_{2} \in Z_{p^{a}}$; and with the action

$$
(m, f, z)^{b}=\left(m^{b}, f^{b}, z\right)
$$

for all $m \in M, f \in M^{*}, z \in Z_{p^{\alpha}}$ and $b \in B$. The details can be found in [3; pages 338-339]. The important features of $M^{\dagger}$ are: the subsets $\{(m, 0,0): m \in M\}$ and $\left\{(0, f, 0): f \in M^{*}\right\}$ are $B$-isomorphic to $M$ and $M^{*}$ (and we usually identify them); $\left\{(0,0, z): z \in Z_{p^{\alpha}}\right\}$ is (additively) isomorphic to $Z_{p^{\alpha}}$ (and we also identify them); and, in the same spirit, for $m \in M, f \in M^{*},[m, f]=f(m)$.

In several places later on it will be important to have the following characterization of $\boldsymbol{M}^{\dagger}$. Here the details suppressed in (2.4) of [3] are made explicit. The result will re-appear in the broader context of realization to be introduced in Section 3.

(2.2) LEMMA. Let $B$ be a group and L a p-group with the following properties.

(i) $L$ has abelian p-subgroups $M, N$ which together generate $L$;

(ii) $L$ is a p-group of class 2 with cyclic centre;

(iii) $L^{\prime}=[M, N]=\zeta_{1}(L) \leqslant \zeta_{1}(L B)$; and

(iv) $M \cap \zeta_{1}(L)=N \cap \zeta_{1}(L)=1$.

Suppose that $\left|\zeta_{1}(L)\right|=p^{\alpha}$ and let $\psi_{0}: \zeta_{1}(L) \rightarrow Z_{p^{\alpha}}^{+}$be an isomorphism.

The function $\theta: N \rightarrow M^{*}$ defined by $\theta_{n}(m)=[m, n] \psi_{0}$ is a B-isomorphism and there is a B-isomorphism $\psi: L \rightarrow M^{\dagger}$ such that

$$
\psi: m \rightarrow(m, 0,0) \text { and } \psi: n \rightarrow\left(0, \theta_{n}, 0\right),
$$

for all $m \in M$ and all $n \in N$.

Proof. That $\theta$ so defined is a $B$-isomorphism is left as an exercise.

Every element of $L$ can be written uniquely in the form $m n z$ ( $m \in M, n \in N$, $\left.z \in \zeta_{1}(L)\right)$. Define $\psi: L \rightarrow M^{\dagger}$ by

$$
\psi: m n z \rightarrow\left(m, \theta_{n}, z \psi_{0}\right), \quad m \in M, n \in N, z \in \zeta_{1}(L) .
$$


Then, writing the group operation in $M$ and $N$ additively where appropriate,

$$
\begin{aligned}
{\left[(m n z)\left(m^{\prime} n^{\prime} z^{\prime}\right)\right] \psi } & =\left[\left(m m^{\prime}\right)\left(n n^{\prime}\right)\left(z z^{\prime}\left[m^{\prime}, n\right]^{-1}\right)\right] \psi \\
& =\left(m+m^{\prime}, n+n^{\prime}, z \psi_{0}+z^{\prime} \psi_{0}-\theta_{n}\left(m^{\prime}\right)\right) \\
& =\left(m, \theta_{n}, z \psi_{0}\right)\left(m^{\prime}, \theta_{n^{\prime}}, z^{\prime} \psi_{0}\right) \\
& =(m n z) \psi\left(m^{\prime} n^{\prime} z^{\prime}\right) \psi ;
\end{aligned}
$$

and since $\psi$ is plainly a $B$-map, and one-to-one and onto, it is a $B$-isomorphism.

The finaly quotation is, in a sense, the crux of [3], and of (4.6) of the present paper. Suppose that $B, B_{i}(i \in I)$ are finite groups and that $M, M_{i}(i \in I)$ are modules for them over (possibly different) rings $Z_{p^{\alpha}}$. Moreover suppose that, for some variety $\mathfrak{B}$,

$$
M=\mathfrak{W}(M B), \quad M_{i}=\mathfrak{W}\left(M_{i} B_{i}\right), \quad i \in I
$$

that $C_{B}(M)=1$; and that $\operatorname{var}\left\{M_{i} B_{i}: i \in I\right\}$ is locally finite.

(2.3) LEMMA. Under the conditions of the last paragraph, if

$$
M B \in \operatorname{var}\left\{M_{i} B_{i}: i \in I\right\},
$$

then

$$
M^{\dagger} B \in \operatorname{var}\left\{M_{i}^{\dagger} B_{i}: i \in I\right\} .
$$

The proof of this result can easily be reconstructed from [3; page 347]. It is this result which enables us to show that $\theta$ is one-to-one if every variety in the set $U$ can be generated by groups of the form $M^{\dagger} B$ : see (4.6).

\section{Symplectic modules}

The group $R$ of (2.1) is of a type familiar in many parts of group theory, its interest lying in the fact that the natural representation of $R$ on $A / A^{\prime}$ is symplectic in the following sense. Suppose that $A^{\prime}$ has order $p^{\alpha}$. It is easy to see that $A / A^{\prime}$ must have exponent precisely $p^{\alpha}$ and may therefore be regarded as a $B$-module over the ring $Z_{p^{\alpha}}$. Commutation in $A$ induces a non-degenerate alternating bilinear form $\langle\rangle:, A / A^{\prime} \times A / A^{\prime} \rightarrow Z_{p^{\alpha}}$ in a well-known way: if $A^{\prime}=\langle z\rangle$ then, for $a_{1}, a_{2} \in A$,

$$
\left[a_{1}, a_{2}\right]=z^{\left\langle a_{1} A^{\prime}, a_{2} A^{\prime}\right\rangle}
$$

the exponent being interpreted modulo $p^{\alpha}$. Since $B$ respects this form its natural representation on the $Z_{p^{\alpha}}$-space $A / A^{\prime}$ is symplectic. 
It is convenient to introduce the following formalism. Let $B$ be a group and $P$ a ring with 1 . A $P B$-module is symplectic if there is a bilinear form $\langle$,$\rangle :$ $M \times M \rightarrow P$ satisfying

(i) $\langle m, m\rangle=0, \quad m \in M$; and

(ii) $\left\langle m_{1}^{b}, m_{2}^{b}\right\rangle=\left\langle m_{1}, m_{2}\right\rangle, \quad m_{1}, m_{2} \in M, b \in B$.

(The reader is referred to Huppert [5] for an account of symplectic modules where, however, the coefficient rings are fields.) In general a bilinear form satisfying (i) is alternating; and one satisfying both (i), (ii) is an invariant scalar product on $M$.

If $N$ is a submodule of a symplectic $P B$-module $M$ then, as usual,

$$
N^{\perp}=\{m \in M:\langle m, n\rangle=0, n \in N\}
$$

is a submodule of $M$. If $N \subseteq N^{\perp}$ then $N$ is an isotropic submodule of $M$, and if $N \cap N^{\perp}=0$ then $N$ is non-degenerate.

A homomorphism $\theta: M \rightarrow N$ between symplectic $P B$-modules is symplectic if $\left\langle m_{1} \theta, m_{2} \theta\right\rangle=\left\langle m_{1}, m_{2}\right\rangle$ for all $m_{1}, m_{2} \in M$. It is usual to call a symplectic isomorphism an isometry.

(3.2) LeMma. Let $P$ be $Z_{p^{a}}$ or a field and let $M$ be a $P B$-module of finite rank.

(i) $M \cong M^{*}$ as $P$-modules.

(ii) Suppose that $M$ is symplectic.

(a) If $U, V$ are submodules of $M$ and $u \in U$, let $\chi_{u}: V \rightarrow P$ be the function $v \mapsto\langle v, u\rangle$. The function $\chi: U \rightarrow V^{*}$ defined by $u \mapsto \chi_{u}$ is a $P B$-homomorphism whose kernel is $U \cap V^{\perp}$.

(b) If $M$ is non-degenerate, $M \cong M^{*}$ as $P B$-modules.

(c) If $V$ is a non-degenerate submodule of $M$, then $M=V \oplus V^{\perp}$.

(iii) Suppose that $P$ is a perfect field of characteristic 2, that $M$ is irreducible and that $M \cong M^{*}$. Then either $M$ admits a non-degenerate scalar product or $M$ is $a$ one-dimensional trivial module.

Note that (iii) is not needed in the present paper. $I$ include it because of its interest as a partial converse to (ii)(b). When the characteristic of $P$ is odd (iii) does not remain true.

Proof of (3.2). (i) This is well-known if $P$ is a field and the case $P=Z_{p^{\alpha}}$ is very similar (see page 338 of [3]). The proof of (ii)(a) is easy and is omitted (it does not, of course rely on these restrictions on $P$ and $M$ ). To prove (ii)(b) let $\chi: M \rightarrow M^{*}$ be defined as in (ii)(a) using $U=V=M$. Since $M$ is non-degenerate $\chi$ is one-to-one and hence is an isomorphism by (i). 
To prove (ii)(c) let $\chi: M \rightarrow V^{*}$ be defined as in (ii)(a). Since $V$ is non-degenerate, $V \cap V^{\perp}=0$. Hence $\chi \mid V$ is one-to-one and therefore, by (i), onto. To each $m \in M$ then there exists $v \in V$ such that $\chi_{m}=\chi_{v}$, which is to say $m-v \in V^{\perp}$. It follows that $M=V \oplus V^{\perp}$.

(iii) Let $\theta: M \rightarrow M^{*}$ be an isomorphism. The function $M \times M \rightarrow P$ defined by $(m, n) \mapsto \theta_{m}(n)+\theta_{n}(m)$ satisfies (3.1) and is bilinear. Hence if it is non-zero we are done. So suppose that, for all $m, n \in M, \theta_{m}(n)+\theta_{n}(m)=0$. Now this means that for all $m, n \in M, \theta_{m+n}(m+n)=\theta_{m}(m)+\theta_{n}(n)$. Also $\theta_{m^{b}}\left(n^{b}\right)=\theta_{m}(n)$ for all $b \in B$. Hence the subset $M_{0}=\left\{m \in M: \theta_{m}(m)=0\right\}$ is a submodule of $M$. Since $M$ is irreducible $M_{0}=0$ or $M_{0}=M$. In the second case the function $M \times M \rightarrow P,(m, n) \mapsto \theta_{m}(n)$ is bilinear and satisfies (3.1) and again we are done. If $\operatorname{dim} M \geqslant 2$ the first case cannot arise. For suppose $M_{0}=0$ and $0 \neq m \in M$. For all $\lambda \in P, \theta_{\lambda m}(\lambda m)=\lambda^{2} \theta_{m}(m)$ and since every non-zero element of $P$ has a square root we may choose $\lambda$ so that $\theta_{\lambda m}(\lambda m)=1$. Hence there are independent elements $m, n \in M$ with $\theta_{m}(m)=\theta_{n}(n)=1$. But then $\theta_{m+n}(m+n)=0$ and $m+n \neq 0$, contradicting $M_{0}=0$. The proof of (3.2) is complete.

We shall need the following constructions of symplectic modules: these, I believe, are not standard. Let $M_{i}(1 \leqslant i \leqslant s)$ be a collection of symplectic $P B$-modules. On the module $\bigoplus_{i=1}^{s} M_{i}$ define a bilinear form by

$$
\langle x, y\rangle=\sum_{i=1}^{s}(-1)^{i-1}\left\langle x \pi_{i}, y \pi_{i}\right\rangle
$$

where $\pi_{i}: \bigoplus_{i=1}^{s} M_{i} \rightarrow M_{i}$ is the natural projection. It is easily checked that this form is bilinear and satisfies (3.1). I denote this symplectic module by

$$
{\underset{i=1}{S}}_{i=1}^{s}
$$

it is more convenient for our purposes in some places than the more usual direct sum (which has a sum, and not an alternating sum, above). However, it is neither commutative nor associative, though

$$
M_{1} Y\left(M_{2} Y M_{3}\right) \cong M_{1} Y M_{2} Y M_{3}
$$

is a convenient right norming.

Next suppose that $M, N$ are $P B$-modules and that $\chi: N \rightarrow M^{*}$ is a homomorphism. We make $M \oplus N$ into a symplectic $P B$-module as follows: define a scalar product on $M \oplus N$ by

$$
\left\langle(m, n),\left(m^{\prime}, n^{\prime}\right)\right\rangle=\chi_{n^{\prime}}(m)-\chi_{n}\left(m^{\prime}\right), \quad m, m^{\prime} \in M, n, n^{\prime} \in N .
$$

Denote this symplectic module by $M \chi N$. Note that the submodules $\{(m, 0): m \in$ $M\}$ and $\{(0, n): n \in N\}$ of $M \chi N$ are isomorphic to $M$ and $N$ respectively and that they are isotropic. 
Much of this paper is concerned with the following problem. Suppose that $M$ is a $Z_{p^{a}} B$-module. When is it possible to find a group $A$, a $p$-group of class 2 with cyclic centre equal to $A^{\prime}$, on which $B$ acts as a group of automorphisms centralizing $A^{\prime}$, and such that $A / A^{\prime}$ is isomorphic to $M$ as a $B$-module? A necessary condition, of course, is that $M$ should admit a scalar product. When $p$ is odd this condition is easily seen to be sufficient: on the set $M \times Z_{p^{a}}$ define the operation

$$
\left(m_{1}, z_{1}\right)\left(m_{2}, z_{2}\right)=\left(m_{1}+m_{2}, z_{1}+z_{2}+\frac{1}{2}\left\langle m_{1}, m_{2}\right\rangle\right)
$$

and the $B$-action $(m, z)^{b}=\left(m^{b}, z\right)$ and check that one has a $B$-group with the desired property.

I now describe this situation in general terms as follows. Let $M$ be a symplectic $P B$-module. Suppose that there is a $B$-group $A$ with the following properties: $A$ has a central subgroup $X$ centralized by $B$ and containing $A^{\prime}$ such that

(i) there is an isomorphism $\phi: X \rightarrow P^{+}$,

(ii) there is a group $B$-isomorphism $\theta: A / X \rightarrow M$, and

(iii) for all $a_{1}, a_{2} \in A$

$$
\left[a_{1}, a_{2}\right] \phi=\left\langle\left(a_{1} X\right) \theta,\left(a_{2} X\right) \theta\right\rangle .
$$

We say then that $A$ realizes $M$.

It will often be convenient to regard $X$ and $A / X$ as $P B$-modules, in fact to use the isomorphisms $\theta, \phi$ to identify $A / X$ with $M$ and $X$ with $P$. In this spirit we have $\left[a_{1}, a_{2}\right]=\left\langle a_{1} X, a_{2} X\right\rangle$ for all $a_{1}, a_{2} \in A$.

The basic results we need about realization follow. In the first result it is only the case $p=2$ which is of interest because of the construction in the antepenultimate paragraph. Then the assumption of solubility is essential because of a result of Greiss [4], a fact pointed out to me by Professor Karl Gruenberg.

(3.4) THeOREM. Let $K$ be a finite soluble group and $M$ a faithful $K$-module over $Z_{p^{\alpha} .}$ Suppose that $O_{p^{\prime}}(K) \neq 1$ and that $\left[M, O_{p^{\prime}}(K)\right]=M$. Then

(i) every $K$-invariant scalar product on $M$ can be realized and

(ii) if $A_{1}, A_{2}$ are $K$-groups realizing isometric scalar products on $M$ then $A_{1} K \cong$ $\mathrm{A}_{2} \mathrm{~K}$.

Proof. We begin by supposing that the image of the scalar product $\langle\rangle:, M \times M \rightarrow Z_{p^{a}}$ is the ideal $\left\langle p^{\gamma}\right\rangle$.

Let $F_{0}$ be the free group of the variety $\mathfrak{A}_{p^{\alpha}} \mathfrak{A}_{p^{\alpha}} \wedge \mathfrak{R}_{2}$ whose rank is the same as that of $M$. There is therefore an onto homomorphism $\alpha_{0}: F_{0} \rightarrow M$ such that

$$
\text { ker } \alpha_{0} \leqslant \Phi\left(F_{0}\right) \text {. }
$$


Since $F_{0}$ is projective there is a subgroup $K_{0}$ of Aut $F_{0}$ and an onto homomorphism $\beta_{0}: K_{0} \rightarrow K$ satisfying

$$
\left(x^{y}\right) \alpha_{0}=\left(x \alpha_{0}\right)^{y \beta_{0}}, \quad x \in F_{0}, y \in K_{0} .
$$

Moreover if $K_{0}$ is chosen to be of minimal order then $\operatorname{ker} \beta_{0} \leqslant \Phi\left(K_{0}\right)$, since any supplement for $\operatorname{ker} \beta_{0}$ in $K_{0}$ would do as well; and since $\operatorname{ker} \beta_{0}$ acts trivially on $F_{0} / \Phi\left(F_{0}\right), \operatorname{ker} \beta_{0}$ is a $p$-group. The Frattini argument then shows that $O_{p^{\prime}}\left(K_{0}\right) \beta_{0}$ $=O_{p^{\prime}}(K)$.

I claim now that there is a homomorphism $\xi: F_{0}^{\prime} \rightarrow Z_{p^{\alpha}}$ satisfying

$$
[x, y] \xi=\left\langle x \alpha_{0}, y \alpha_{0}\right\rangle, \quad x, y \in F_{0} .
$$

For, let $\left\{x_{1}, x_{2}, \ldots, x_{m}\right\}$ be a free generating set for $F_{0}$. Then every element of $F_{0}^{\prime}$ has a unique representation in the form

$$
\begin{array}{r}
{\left[x_{2}, x_{1}\right]^{\lambda_{21}}\left[x_{3}, x_{1}\right]^{\lambda_{31}} \cdots\left[x_{m}, x_{1}\right]^{\lambda_{m 1}}\left[x_{3}, x_{2}\right]^{\lambda_{32}}} \\
\cdots\left[x_{m}, x_{2}\right]^{\lambda_{m 2}} \cdots\left[x_{m}, x_{m-1}\right]^{\lambda_{m m-1}},
\end{array}
$$

where $0 \leqslant \lambda_{i j} \leqslant p^{\alpha}-1$ for all $i, j$. The existence of such an expression is obvious, and uniqueness is easy: if such an expression is 1 in $F_{0}$ then the endomorphism of $F_{0}$ defined by

$$
x_{i} \mapsto x_{i}, \quad x_{j} \mapsto x_{j}, \quad x_{k} \mapsto 1, \quad k \notin\{i, j\},
$$

yields $\left[x_{i}, x_{j}\right]^{\lambda_{i j}}=1$ whence $p^{\alpha} \mid \lambda_{i j}$ or $\lambda_{i j}=0$, and this for every pair $i, j$ with $i>j$. This ensures the existence of $\xi$. Note that the image of $\xi$ is $\left\langle p^{\gamma}\right\rangle$ because of the assumption in the first sentence of this proof. Note also that for all $k \in K_{0}$,

$$
\begin{aligned}
{\left[x^{k}, y^{k}\right] \xi } & =\left\langle x^{k} \alpha_{0}, y^{k} \alpha_{0}\right\rangle=\left\langle\left(x \alpha_{0}\right)^{k \beta_{0}},\left(y \alpha_{0}\right)^{k \beta_{0}}\right\rangle \\
& =\left\langle x \alpha_{0}, y \alpha_{0}\right\rangle=[x, y] \xi,
\end{aligned}
$$

so that $\xi$ is a $K_{0}$-homomorphism if we regard $Z_{p^{\alpha}}$ as a trivial $K_{0}$-module.

A final preliminary observation before constructing a realizing group: $O_{p^{\prime}}\left(K_{0}\right)$ has no fixed points in $F_{0} / F_{0}^{\prime}$. For, if $U / F_{0}^{\prime}$ is the fixed point space of $O_{p^{\prime}}\left(K_{0}\right)$ in $F_{0} / F_{0}^{\prime}$ then $U \leqslant \operatorname{ker} \alpha_{0} / F_{0}^{\prime}$ since $O_{p^{\prime}}\left(K_{0}\right)$ has no fixed points in $M$. But $U$ has a complement in $F_{0} / F_{0}^{\prime}$ by Fitting's Lemma, contradicting ker $\alpha_{0} \leqslant \Phi\left(F_{0}\right)$, unless $U / F_{0}^{\prime}=1$.

Now, modulo ker $\xi$, Fitting's Lemma ensures that $F_{0}^{\prime}$ has a complement admitting $K_{0}$ in $\operatorname{ker} \alpha_{0}$. Consequently, there exists in $\operatorname{ker} \alpha_{0}$ a subgroup $C$ with the properties: $C \unlhd F_{0} K_{0}$, ker $\alpha_{0}=F_{0}^{\prime} C$ and $F_{0}^{\prime} \cap C=\operatorname{ker} \xi$.

Consider the group $F_{0} / C$ and call it $A_{0}$ : it will turn out to be almost a realizing group for $M$.

To see this, let $X_{0}=A_{0}^{\prime}$ and define $\phi: X_{0} \rightarrow Z_{p^{\alpha}}$ by

$$
\phi_{0}: z C \mapsto z \xi, \quad z \in F_{0}^{\prime},
$$


and $\theta_{0}: A_{0} / X_{0} \rightarrow M$ by

$$
\theta_{0}:(x C)\left(F_{0}^{\prime} C / C\right) \mapsto x \alpha_{0}, \quad x \in F_{0} .
$$

Then for all $x, y \in F_{0}$,

$$
\begin{aligned}
{[x C, y C] \phi_{0} } & =[x, y] C \phi_{0}=[x, y] \xi=\left\langle x \alpha_{0}, y \alpha_{0}\right\rangle \\
& =\left\langle(x C)\left(F_{0}^{\prime} C / C\right) \theta_{0},(y C)\left(F_{0}^{\prime} C / C\right) \theta_{0}\right\rangle \\
& =\left\langle(x C) X_{0} \theta_{0},(y C) X_{0} \theta_{0}\right\rangle .
\end{aligned}
$$

Next, we show that $K$ acts on $A_{0}$. For let

$$
L=\left\{l \in \text { Aut } F_{0}: \operatorname{ker} \alpha_{0} \text { admits } l,\left[F_{0}, l\right] \leqslant \operatorname{ker} \alpha_{0} \text { and } l \in \mathbf{C}_{\text {Aut } F_{0}}\left(O_{p^{\prime}}\left(K_{0}\right)\right)\right\} \text {. }
$$

Then $K_{0}$ normalizes $L$, and $\operatorname{ker} \beta_{0} \leqslant L$. Also

$$
\left[F_{0}, L\right] \leqslant F_{0}^{\prime} \cap \operatorname{ker} \alpha_{0} .
$$

To see this, note first that

$$
\begin{aligned}
{\left[F_{0}^{\prime}, L\right] } & =\left[F_{0}, F_{0}, L\right] \leqslant\left[F_{0}, F_{0}, L \operatorname{ker} \alpha_{0}\right] \\
& \leqslant\left[F_{0}, L \operatorname{ker} \alpha_{0}, F_{0}\right]\left[L \operatorname{ker} \alpha_{0}, F_{0}, F_{0}\right],
\end{aligned}
$$

by the three subgroup lemma, whence $\left[F_{0}^{\prime}, L\right] \leqslant F_{0}^{\prime} \cap \operatorname{ker} \alpha_{0}$; and then it follows that there is for each $l \in L$ an $O_{p^{\prime}}\left(K_{0}\right)$-homomorphism $F_{0} / F_{0}^{\prime} \rightarrow F_{0}^{\prime} / F_{0}^{\prime} \cap$ ker $\alpha_{0}$ defined by $x F_{0}^{\prime} \mapsto[x, l]$ which, of course, must be zero since the composition factors of the $O_{p^{\prime}}\left(K_{0}\right)$-module $F_{0} / F_{0}^{\prime}$ are all non-trivial whilst $F_{0}^{\prime} / F_{0}^{\prime} \cap$ ker $\alpha_{0}$ is trivial. Therefore (3.4.1) is confirmed. It follows that $\operatorname{ker} \beta_{0} \unlhd A_{0} K_{0}$ and so it may be factored out leaving $K$ acting on $A_{0}$.

The group $A_{0}$ now satisfies the requirements of a realizing group for $M$ except that $\phi_{0}: X_{0} \rightarrow Z_{p^{\mathrm{a}}}$ whilst being one-to-one is not necessarily an isomorphism. But a central product of $A_{0}$ and $Z_{p^{\alpha}}$ will provide a realizing group for $M$. To be precise let $N=\left\{\left(z^{-1}, z \phi_{0}\right): z \in A_{0}^{\prime}\right\}$ and put $A=\left(A_{0} \times Z_{p^{\alpha}}\right) / N$; then it is clear that $A$ realizes $M$.

We now take up the question of uniqueness. To begin, suppose that $A_{1}$ is a realizing group for $M$ with subgroup $X_{1}$ and isomorphisms $\theta_{1}, \phi_{1}$ satisfying the definition of realization. Since $O_{p^{\prime}}(K)$ has no fixed points in $A_{1} / X_{1}$ it follows that there is a unique, $K$-admissible subgroup $A_{2} / A_{1}^{\prime}$ of $A_{1} / A_{1}^{\prime}$ such that

$$
A_{1} / A_{1}^{\prime}=A_{2} / A_{1}^{\prime} \times X_{1} / A_{1}^{\prime}
$$

this because of Fitting's Lemma. The group $A_{2}$ almost realizes $M$ in the sense that there exists an isomorphism $\theta_{2}: A_{2} / A_{2}^{\prime} \rightarrow M$ and a monomorphism $\phi_{2}: A_{2}^{\prime} \rightarrow Z_{p^{\alpha}}$ such that

$$
\left[a_{1} A_{2}^{\prime}, a_{2}, A_{2}^{\prime}\right] \phi_{2}=\left\langle\left(a_{1} A_{2}^{\prime}\right) \theta_{2},\left(a_{2} A_{2}^{\prime}\right) \theta_{2}\right\rangle, \quad a_{1}, a_{2} \in A_{2} .
$$

Of course $A_{1}$ is a central product of $A_{2}$ and $X_{1}$ amalgamating all of $A_{2}^{\prime}$. 
Conversely, if we have a group $A_{2}$ with the properties described we can construct a realizing group for $M$ as such a central product. Moreover, since all possible ways of amalgamating $A_{2}^{\prime}$ into $Z_{p^{\alpha}}$ are permuted transitively by the automorphisms of $Z_{p^{\alpha}}$ all such realizations $A_{1}$ of $M$ constructed from $A_{2}$ are $K$-isomorphic.

Hence to conclude the proof we need to show that if $A_{2}, A_{3}$ are $K$-groups satisfying (3.4.2) then $A_{2} K \cong A_{3} K$. Since $F_{0}$ is projective in $\mathfrak{A}_{p^{a}} \mathfrak{A}_{p^{\alpha}} \wedge \mathfrak{N}_{2}$ there exist onto homomorphisms $\alpha_{i}: F_{0} \rightarrow A_{i}(i=2,3)$ and subgroups $K_{i}(i=2,3)$ of Aut $F_{0}$ with onto homomorphisms $\beta_{i}: K_{i} \rightarrow K$ satisfying

$$
\left(x^{y}\right) \alpha_{i}=\left(x \alpha_{i}\right)^{y \beta_{i}}, \quad x \in F_{0}, y \in K_{i}, i=2,3,
$$

and

$$
\left(x \alpha_{i}\right) A_{i}^{\prime} \theta_{i}=x \alpha_{0}, \quad x \in F_{0}, i=2,3 .
$$

Moreover, as before in the case of $K_{0}$, we may suppose that for $i=2,3$ $\operatorname{ker} \beta_{i} \leqslant \Phi\left(K_{i}\right)$, that $\operatorname{ker} \beta_{i}$ is a $p$-group and that $O_{p^{\prime}}\left(K_{i}\right) \beta_{i}=O_{p^{\prime}}(K)$.

We investigate the connexion between $K_{2}$ and $K_{3}$. First

$$
\left(\operatorname{ker} \alpha_{i}\right) F_{0}^{\prime}=\operatorname{ker} \alpha_{0}, \quad i=2,3 \text {. }
$$

For, if $x \in \operatorname{ker} \alpha_{i}$, that is if $x \alpha_{i}=1$, then by (3.4.3) $x \alpha_{0}=1$ so $\operatorname{ker} \alpha_{i} \subseteq \operatorname{ker} \alpha_{0}$. Also if $y \in \operatorname{ker} \alpha_{0}$ then $y \alpha_{i} \in A_{i}^{\prime}$ by (3.4.3) and hence for some $z \in F_{0}^{\prime}, z \alpha_{i}=y \alpha_{i}$ or $y z^{-1} \in \operatorname{ker} \alpha_{i}$. Therefore (3.4.4) is proved.

A corollary of this is that ker $\alpha_{0}$ admits $K_{2}$ and $K_{3}$. Also

$$
\operatorname{ker} \alpha \cap F_{0}^{\prime}=\operatorname{ker} \alpha_{3} \cap F_{0}^{\prime} \text {. }
$$

For, if $x, y \in F_{0}$ then

$$
\begin{aligned}
{[x, y] \alpha_{2} \phi_{2} } & =\left[x \alpha_{2}, y \alpha_{2}\right] \phi_{2}=\left\langle\left(x \alpha_{2}\right) A_{2}^{\prime} \theta_{2},\left(y \alpha_{2}\right) A_{2}^{\prime} \theta_{2}\right\rangle \\
& =\left\langle x \alpha_{0}, y \alpha_{0}\right\rangle=\left\langle\left(x \alpha_{3}\right) A_{3}^{\prime} \theta_{3},\left(y \alpha_{3}\right) A_{2}^{\prime} \theta_{3}\right\rangle \\
& =\left[x \alpha_{3}, y \alpha_{3}\right] \phi_{3}=[x, y] \alpha_{3} \phi_{3}
\end{aligned}
$$

whence, on restriction to $F_{0}^{\prime}, \alpha_{2} \phi_{2}=\alpha_{3} \phi_{3}$, which ensures (3.4.5).

Next for each $k_{2} \in K_{2}$ there exists $k_{3} \in K_{3}$ such that $k_{2}^{-1} k_{3}$ acts trivially on $F_{0} /$ ker $\alpha_{0}$. For given $k_{2} \in K_{2}$ choose $k_{3}$ to satisfy $k_{2} \beta_{2}=k_{3} \beta_{3}$. Then for all $x \in F_{0}$

$$
\begin{aligned}
\left(x^{k_{2}^{-1} k_{3}}\right) \alpha_{0} & =\left(x^{k_{2}^{-1} k_{3}} \alpha_{3}\right) A_{3}^{\prime} \theta_{3}=\left(x^{k_{2}^{-1}} \alpha_{3}\right)^{k_{3} \beta_{3}} A_{3}^{\prime} \theta_{3} \\
& =\left(\left(x^{k_{2}^{-1}} \alpha_{3}\right) A_{3}^{\prime} \theta_{3}\right)^{k_{2} \beta_{2}}=\left(x^{k_{2}^{-1}} \alpha_{0}\right)^{k_{2} \beta_{2}} \\
& =\left(x^{k_{2}^{-1}} \alpha_{2}\right)^{k_{2} \beta_{2}} A_{2}^{\prime} \theta_{2}=\left(x \alpha_{2}\right) A_{2}^{\prime} \theta_{2}=x \alpha_{0},
\end{aligned}
$$

so $k_{2}^{-1} k_{3}$ acts trivially on $F_{0} / \operatorname{ker} \alpha_{0}$. Consequently, if $L$ is the subgroup of Aut $F_{0}$ of all automorphisms admitted by $\operatorname{ker} \alpha_{0}$ and acting trivially on $F_{0} / \operatorname{ker} \alpha_{0}$ then 
$K_{2} L=K_{3} L$, and, of course, $K_{i} \cap L=\operatorname{ker} \beta_{i}, i=2,3$. Hence, modulo $L, O_{p^{\prime}}\left(K_{2}\right)$ and $O_{p^{\prime}}\left(K_{3}\right)$ are equal and therefore for some $l \in L$,

$$
O_{p^{\prime}}\left(K_{3}\right)^{l}=O_{p^{\prime}}\left(K_{2}\right) \text {. }
$$

Since conjugation by $l$ in $F_{0} K_{3}$ induces an isomorphism of the group $A_{3} K$ we may, without loss of generality, assume that $l=1$. For convenience write $N=$ $O_{p^{\prime}}\left(K_{2}\right)\left(=O_{p^{\prime}}\left(K_{3}\right)\right)$ and $L_{0}=\mathbf{C}_{L}(N)$. Then

$$
\mathbf{N}_{K_{2} L}(N)=K_{i} L_{0}, \quad i=2,3 .
$$

Now it follows from (3.4.6) that ker $\alpha_{0}$ is central in $F_{0}$ modulo ker $\alpha_{2} \cap F_{0}^{\prime}$. Hence Fitting's Lemma yields the existence of normal subgroups $C_{i}$ of $F_{0}$ admitting $K_{i}$ and such that

$$
\operatorname{ker} \alpha_{i}=C_{i}\left(F_{0}^{\prime} \cap \operatorname{ker} \alpha_{i}\right) \text { and } C_{i} \cap F_{0}^{\prime}=F_{0}^{\prime} \cap \operatorname{ker} \alpha_{i} .
$$

However, $C_{i}$ is then a complement for $F_{0}^{\prime}$ in ker $\alpha_{0}$ modulo $F_{0}^{\prime} \cap$ ker $\alpha_{2}$ admitting $O_{p^{\prime}}\left(K_{2}\right)$. Since there is only one such complement $C_{2}=C_{3}=C$, say. It follows that

$$
\operatorname{ker} \alpha_{2}=\operatorname{ker} \alpha_{3} .
$$

Next, let $L_{1}=\left\{l \in L_{0}: \operatorname{ker} \alpha_{2}\right.$ admits $\left.l\right\}$. Then since $K_{2} L_{0}=K_{3} L_{0}$ it follows that $K_{2}, K_{3}$ normalize $L_{1}$ and

$$
K_{2} L_{1}=K_{3} L_{1} \quad \text { and } \quad K_{i} \cap L_{1}=\operatorname{ker} \beta_{i}, \quad i=2,3 .
$$

Moreover, it follows from (3.4.1) that

$$
\left[F_{0}, L_{1}\right] \leqslant \operatorname{ker} \alpha_{2} \text {. }
$$

Now $\beta_{i}: K_{i} \rightarrow K$ may be extended to a homomorphism from $K_{i} L_{1} \rightarrow K$ and we shall call this $\beta_{i}$ also:

$$
\left(k_{i} l_{1}\right) \rightarrow k_{i} \beta_{i}, \quad k_{i} \in K_{i}, l_{1} \in L_{i}, i=2,3,
$$

is well defined since $K_{i} \cap L_{1}=\operatorname{ker} \beta_{i}$. Then there exist onto homomorphisms $\gamma_{i}$ : $F_{0} K_{i} L_{1} \rightarrow A_{i} K$ defined by

$$
(f x) \gamma_{i}=\left(f \alpha_{i}\right)\left(x \beta_{i}\right), \quad f \in F_{0}, x \in K_{i} L,
$$

and both have kernel equal to $L_{1}$ ker $\alpha_{2}$. Consequently, the images of $\gamma_{2}$ and $\gamma_{3}$ are isomorphic which is to say $A_{2} K \cong A_{3} K$, as required.

(3.5) Lemma. (i) Every symplectic submodule of a realizable symplectic PB-module is realizable.

(ii) If $M_{i}(1 \leqslant i \leqslant s)$ are realizable symplectic $P B$-modules so is $Y_{i=1}^{s} M_{i}$ and $a$ realizing group for it is a central product amalgamating $P$ of realizing groups for the $\boldsymbol{M}_{i}$.

(iii) If $\chi: N \rightarrow M^{*}$ is a homomorphism of $P B$-modules $M, N$ then $M \chi N$ is realizable. 
Proof. The proof of (i) is easy and is omitted. To prove (ii) it suffices, by (3.3), to assume $s=2$. Let $A_{1}, A_{2}$ be groups realizing $M_{1}, M_{2}$. In what follows the notation in the definition of realizing group is used with subscripts to refer to $A_{1}$ and $A_{2}$.

Let $A_{0}=A_{1} \times A_{2}$ as $B$-group and consider the subgroup

$$
U=\left\{\left(x, x \phi_{1} \phi_{2}^{-1}\right): x \in X_{1}\right\} .
$$

I claim that $A=A_{0} / U$ realizes $M_{1} Y M_{2}$.

Put $X=\left(X_{1} \times X_{2}\right) / U$. Since every element $z$ of $X_{1} \times X_{2}$ can be written uniquely in the form

$$
z=(x, 0)+u
$$

where $x \in X_{1}$ and $u \in U$ we may define an isomorphism $\phi: X \rightarrow P^{+}$by

$$
(z U) \phi=x \phi_{1} \text {. }
$$

Now define $\theta: A_{0} /\left(X_{1} \times X_{2}\right)(\cong A / X) \rightarrow M_{1} Y M_{2}$ by

$$
\theta:\left(a_{1}, a_{2}\right)\left(X_{1} \times X_{2}\right) \rightarrow\left(\left(a_{1} X_{1}\right) \theta_{1},\left(a_{2} X_{2}\right) \theta_{2}\right), \quad a_{1} \in A_{1}, a_{2} \in A_{2} .
$$

Clearly, $\theta$ is a $B$-isomorphism and we need only check condition (iii). For all $a_{1}, a_{1}^{\prime} \in A_{1}, a_{2}, a_{2}^{\prime} \in A_{2}$,

$$
\begin{aligned}
{\left[\left(a_{1}, a_{2}\right) U,\left(a_{1}^{\prime}, a_{2}^{\prime}\right) U\right] \phi } & =\left(\left(\left[a_{1}, a_{1}^{\prime}\right],\left[a_{2}, a_{2}^{\prime}\right]\right) U\right) \phi \\
& =\left(\left(\left[a_{1}, a_{1}^{\prime}\right]-\left[a_{2}, a_{2}^{\prime}\right] \phi_{2} \phi_{1}^{-1}, 0\right) U\right) \phi \\
& =\left(\left[a_{1}, a_{1}^{\prime}\right]-\left[a_{2}, a_{2}^{\prime}\right] \phi_{2} \phi_{1}^{-1}\right) \phi_{1} \\
& =\left[a_{1}, a_{1}^{\prime}\right] \phi_{1}-\left[a_{2}, a_{2}^{\prime}\right] \phi_{2} \\
& =\left\langle\left(a_{1} X_{1}\right) \theta_{1},\left(a_{1}^{\prime} X_{1}\right) \theta_{1}\right\rangle-\left\langle\left(a_{2} X_{2}\right) \theta_{2},\left(a_{2}^{\prime} X_{2}\right) \theta_{2}\right\rangle \\
& =\left\langle\left(\left(a_{1} X_{1}\right) \theta_{1},\left(a_{2} X_{2}\right) \theta_{2}\right),\left(\left(a_{1}^{\prime} X_{1}\right) \theta_{1},\left(a_{2}^{\prime} X_{2}\right) \theta_{2}\right)\right\rangle \\
& =\left\langle\left(\left(a_{1}, a_{2}\right)\left(X_{1} \times X_{2}\right)\right) \theta,\left(\left(a_{1}^{\prime}, a_{2}^{\prime}\right)\left(X_{1} \times X_{2}\right)\right) \theta\right\rangle,
\end{aligned}
$$

as required.

To prove (iii) define a product on $M \times N \times P$ by

$$
\left(m_{1}, n_{1}, r_{1}\right)\left(m_{2}, n_{2}, r_{2}\right)=\left(m_{1}+m_{2}, n_{1}+n_{2}, r_{1}+r_{2}-\chi_{n_{1}}\left(m_{2}\right)\right),
$$

for $m_{i} \in M, n_{i} \in N, r_{i} \in P$. It is easy to check that this is a group operation. Denote this group by $A$. It is a $B$-group under the action

$$
(m, n, r)^{b}=\left(m^{b}, n^{b}, r\right), \quad m \in M, n \in N, r \in P, b \in B .
$$

Let $X=\{(0,0, r): r \in P\}$. Plainly $\phi:(0,0, r) \rightarrow r$ is an isomorphism $X \rightarrow P^{+}$. Also define $\theta: A / X \rightarrow M \chi N$ by

$$
\theta:(m, n, r) X \rightarrow(m, n)
$$


a $B$-isomorphism. Then

$$
\begin{aligned}
{\left[\left(m_{1}, n_{1}, r_{1}\right),\left(m_{2}, n_{2}, r_{2}\right)\right] \phi } & =\left(0,0, \chi_{n_{2}}\left(m_{1}\right)-\chi_{n_{1}}\left(m_{2}\right)\right) \phi \\
& =\chi_{n_{2}}\left(m_{1}\right)-\chi_{n_{1}}\left(m_{2}\right) \\
& =\left\langle\left(m_{1}, n_{1}\right),\left(m_{2}, n_{2}\right)\right\rangle \\
& =\left\langle\left(m_{1}, n_{1}, r_{1}\right) \theta,\left(m_{2}, n_{2}, r_{2}\right) \theta\right\rangle
\end{aligned}
$$

so $A$ does realize $M \chi N$. This completes the proof of (3.5).

We will denote the group realizing $M \chi N$ by $\chi^{\dagger}$. It should be noted that the group $M^{\dagger}$ introduced in Section 2 is a special case of this construction: if $\iota$ : $M^{*} \rightarrow M^{*}$ is the identity map then $M \iota M^{*} \cong \iota^{\dagger}$.

The next result is a technical one. Later on we use it to make the first reduction in analysing the groups $R$ of (2.1).

(3.6) Lemma. Let $M$ be a symplectic PB-module which has sub-modules $M_{1}, M_{2}$ with $M=M_{1}+M_{2}$. Let $\chi: M_{2} \rightarrow M_{1}^{*}$ be defined as in (3.2)(ii)(a). Then there exist exact sequences of $P B$-modules

(i) $0 \rightarrow M_{1} \chi M_{2} \rightarrow M Y M Y M$,

(ii)

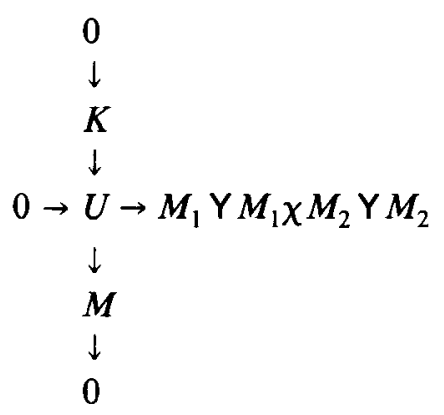

where all the homomorphisms are symplectic and where $K \subseteq U^{\perp}$.

Proof. (i) Define $\theta: M_{1} \chi M_{2} \rightarrow M Y M Y M$ by

$$
\left(m_{1}, m_{2}\right) \theta=\left(m_{1}, m_{1}-m_{2}, m_{2}\right), \quad m_{1} \in M_{1}, m_{2} \in M_{2} .
$$

Clearly, $\theta$ is a one-to-one homomorphism and it is symplectic because, for all $\left(m_{1}, m_{2}\right),\left(m_{1}^{\prime}, m_{2}^{\prime}\right) \in M_{1} \chi M_{2}$,

$$
\begin{aligned}
\left\langle\left(m_{1}, m_{2}\right) \theta,\left(m_{1}^{\prime}, m_{2}^{\prime}\right) \theta\right\rangle & =\left\langle\left(m_{1}, m_{1}-m_{2}, m_{2}\right),\left(m_{1}^{\prime}, m_{1}^{\prime}-m_{2}^{\prime}, m_{2}^{\prime}\right)\right\rangle \\
& =\left\langle m_{1}, m_{1}^{\prime}\right\rangle-\left\langle m_{1}-m_{2}, m_{1}^{\prime}-m_{2}^{\prime}\right\rangle+\left\langle m_{2}, m_{2}^{\prime}\right\rangle \\
& =\left\langle m_{1}, m_{2}^{\prime}\right\rangle-\left\langle m_{1}^{\prime}, m_{2}\right\rangle=\chi_{m_{2}^{\prime}}\left(m_{1}\right)-\chi_{m_{2}}\left(m_{1}^{\prime}\right) \\
& =\left\langle\left(m_{1}, m_{2}\right),\left(m_{1}^{\prime}, m_{2}^{\prime}\right)\right\rangle .
\end{aligned}
$$


(ii) Let $U$ be the submodule of $M_{1} Y M_{1} \chi M_{2} Y M_{2}$ defined by

$$
U=\left\{\left(m_{1},\left(m_{1}, m_{2}\right), m_{2}\right): m_{1} \in M_{1}, m_{2} \in M_{2}\right\} .
$$

Define a function $\phi: U \rightarrow M$ by

$$
\left(m_{1},\left(m_{1}, m_{2}\right), m_{2}\right) \phi=m_{1}-m_{2} \text {. }
$$

Clearly, $\phi$ is a $P B$-homomorphism. It is symplectic because

$$
\begin{aligned}
\left\langle\left(m_{1},\left(m_{1}, m_{2}\right), m_{2}\right) \phi,\right. & \left.\left(m_{1}^{\prime},\left(m_{1}^{\prime}, m_{2}^{\prime}\right), m_{2}^{\prime}\right) \phi\right\rangle=\left\langle m_{1}-m_{2}, m_{1}^{\prime}-m_{2}^{\prime}\right\rangle \\
& =\left\langle m_{1}, m_{1}^{\prime}\right\rangle-\left(\left\langle m_{1}, m_{2}^{\prime}\right\rangle-\left\langle m_{1}^{\prime}, m_{2}\right\rangle\right)+\left\langle m_{2}, m_{2}^{\prime}\right\rangle \\
& =\left\langle m_{1}, m_{1}^{\prime}\right\rangle-\left(\chi_{m_{2}^{\prime}}\left(m_{1}\right)-\chi_{m_{2}}\left(m_{1}^{\prime}\right)\right)+\left\langle m_{2}, m_{2}^{\prime}\right\rangle \\
& =\left\langle\left(m_{1},\left(m_{1}, m_{2}\right), m_{2}\right),\left(m_{1}^{\prime},\left(m_{1}^{\prime}, m_{2}^{\prime}\right), m_{2}^{\prime}\right)\right\rangle
\end{aligned}
$$

as required.

Finally, $\operatorname{ker} \phi=\left\{(m,(m, m), m): m \in M_{1} \cap M_{2}\right\}$ and, for all $m \in M_{1} \cap M_{2}$, $m_{1} \in M_{1}, m_{2} \in M_{2}$

$$
\begin{aligned}
\left\langle(m,(m, m), m),\left(m_{1},\left(m_{1}, m_{2}\right), m_{2}\right)\right\rangle & \\
& =\left\langle m, m_{1}\right\rangle-\left(\chi_{m_{2}}(m)-\chi_{m}\left(m_{1}\right)\right)+\left\langle m, m_{2}\right\rangle \\
& =\left\langle m, m_{1}\right\rangle-\left\langle m, m_{2}\right\rangle-\left\langle m, m_{1}\right\rangle+\left\langle m, m_{2}\right\rangle=0 .
\end{aligned}
$$

Hence $\operatorname{ker} \phi \subseteq U^{\perp}$ as required. Since $\phi$ is onto we are done.

(3.7) Lemma. Suppose that $P$ is $Z_{p^{\alpha}}$ or that $P$ is a field and let $M$ be a non-degenerate symplectic $P B$-module. Let $\chi: M \rightarrow M^{*}$ be the induced isomorphism with $\chi_{m}\left(m^{\prime}\right)=\left\langle m^{\prime}, m\right\rangle$ ensured by (3.2). Then

(i) $M \chi M$ and $M \iota M^{*}$ are isometric, and

(ii) If End $M$ contains a symplectic endomorphism $e$ such that $1-e$ is invertible then $M \chi M$ and $M Y M$ are isometric.

Proof. (i) This is almost a tautology: it is easy to verify that the function $(m, n) \rightarrow\left(m, \chi_{n}\right)$ is an isometry.

(ii) Define $\phi: M \chi M \rightarrow M Y M$ by

$$
\left(m_{1}, m_{2}\right) \phi=\left(m_{1}+(1-e)^{-1} m_{2}, m_{1}+e(1-e)^{-1} m_{2}\right), \quad m_{1}, m_{2} \in M .
$$


It is easy to check that $\phi$ is a $P B$-isomorphism. It is, moreover, an isometry:

$$
\begin{aligned}
\left\langle\left(m_{1}, m_{2}\right) \phi,\left(m_{1}^{\prime}, m_{2}^{\prime}\right) \phi\right\rangle=\left\langle m_{1}+(1-e)^{-1} m_{2}, m_{1}^{\prime}+(1-e)^{-1} m_{2}^{\prime}\right\rangle & -\left\langle m_{1}+e(1-e)^{-1} m_{2}, m_{1}^{\prime}+e(1-e)^{-1} m_{2}^{\prime}\right\rangle \\
= & \left(\left\langle\left(m_{1}, m_{1}^{\prime}\right)\right\rangle+\left\langle m_{1},(1-e)^{-1} m_{2}^{\prime}\right\rangle+\left\langle(1-e)^{-1} m_{2}, m_{1}^{\prime}\right\rangle\right. \\
& \left.+\left\langle(1-e)^{-1} m_{2},(1-e)^{-1} m_{2}^{\prime}\right\rangle\right) \\
& -\left(\left\langle m_{1}, m_{1}^{\prime}\right\rangle+\left\langle m_{1}, e(1-e)^{-1} m_{2}^{\prime}\right\rangle+\left\langle e(1-e)^{-1} m_{2}, m_{1}^{\prime}\right\rangle\right. \\
& \left.+\left\langle e(1-e)^{-1} m_{2}, e(1-e)^{-1} m_{2}^{\prime}\right\rangle\right) \\
= & \left\langle m_{1},(1-e)(1-e)^{-1} m_{2}^{\prime}\right\rangle+\left\langle(1-e)(1-e)^{-1} m_{2}, m_{1}^{\prime}\right\rangle \\
= & \left\langle m_{1}, m_{2}^{\prime}\right\rangle-\left\langle m_{1}^{\prime}, m_{2}\right\rangle=\left\langle\left(m_{1}, m_{2}\right),\left(m_{1}^{\prime}, m_{2}^{\prime}\right)\right\rangle .
\end{aligned}
$$

(3.8) Lemma. Let $P$ be a finite field of characteristic 2, let $B$ be a finite group and let $N$ be an irreducible $P B$-module.

(i) If $N_{1}, N_{2}$ are non-degenerate symplectic PB-modules isomorphic to $N$ then $N_{1}$ and $\mathrm{N}_{2}$ are isometric.

(ii) The following conditions are equivalent.

(a) $N_{\iota} N^{*}$ has an irreducible non-isotropic submodule.

(b) $N$ admits a non-degenerate scalar product and End $N$ contains a non-identity isometry.

(c) $N$ admits a non-degenerate scalar product and $N_{\iota} N^{*}$ is isometric to $N Y N$.

Proor. (i) Write $N_{0}=N_{1} Y N_{2}$ and let $U$ be an irreducible submodule of $N_{0}$. Then $U \cong N$. If $U$ is non-isotropic then $N_{0}=U \oplus U^{\perp}$ by (3.2), $U^{\perp} \cong N$ and $U^{\perp}$ is non-isotropic since $N_{0}$ is non-degenerate. Now every irreducible submodule of $N_{0}$, other than $N_{2}$, has the form

$$
\left\{(n, n \alpha): n \in N_{1}\right\}
$$

for some $\alpha \in \operatorname{hom}\left(N_{1}, N_{2}\right)$. But $\left|\operatorname{hom}\left(N_{1}, N_{2}\right)\right|=\mid$ End $N \mid$, a power of 2 since End $N$ is a finite field of characteristic 2 . Hence $N_{0}$ has an odd number of irreducible submodules. In consequence at least one irreducible submodule of $N_{0}$ is isotropic. This means that for some $0 \neq \alpha \in \operatorname{hom}\left(N_{1}, N_{2}\right)$,

$$
\begin{aligned}
0 & =\left\langle\left(n_{1}, n_{2} \alpha\right),\left(n_{2}, n_{2}, \alpha\right)\right\rangle, \quad n_{1}, n_{2} \in N_{1}, \\
& =\left\langle n_{1}, n_{2}\right\rangle+\left\langle n_{1} \alpha, n_{2} \alpha\right\rangle
\end{aligned}
$$

so

$$
\left\langle n_{1}, n_{2}\right\rangle=\left\langle n_{1} \alpha, n_{2} \alpha\right\rangle, \quad n_{1}, n_{2} \in N_{1},
$$

the characteristic being 2 . Hence $\alpha$ is an isometry. 
(ii) Suppose that $V$ is an irreducible non-isotropic submodule of $N \iota N^{*}$. This means that $V$ admits a non-degenerate scalar product and hence that $V \cong V^{*}$ by (3.2). Since $N^{*}$ is irreducible it follows that $V \cong N$ or $V \cong N^{*}$. In any case $V \cong N$. Also, $N_{\iota} N^{*}=V \oplus V^{\perp}$ by (3.2) so $V^{\perp} \cong N$ also and $V^{\perp}$ is non-isotropic since $N \iota N^{*}$ is non-degenerate.

Now $N=\{v+v \alpha: v \in V\}$ for some $0 \neq \alpha \in \operatorname{hom}\left(V, V^{\perp}\right)$. Since $N$ is isotropic we deduce that for all $v, v^{\prime} \in V$

$$
0=\left\langle v+v \alpha, v^{\prime}+v^{\prime} \alpha\right\rangle=\left\langle v, v^{\prime}\right\rangle+\left\langle v \alpha, v^{\prime} \alpha\right\rangle,
$$

and therefore, $\left\langle v, v^{\prime}\right\rangle=\left\langle v \alpha, v^{\prime} \alpha\right\rangle$ since the characteristic is 2. Thus $\alpha$ is an isometry. Similarly, there exists an isometry $\beta \in \operatorname{hom}\left(V, V^{\perp}\right)$, different from zero and from $\alpha$ such that $N^{*}=\{v+v \beta: v \in V\}$. But then $\alpha \beta^{-1}$ is an isometry on $V$ and $1 \neq \alpha \beta^{-1} \in$ End $V$. Since $V \cong N$ this completes the proof that (a) implies (b).

That (b) implies (c) follows from (3.7) and (c) implies (a) is obvious.

\section{Proof of (1.1)}

Here we establish the existence of the map $\theta: \mathcal{Q} \rightarrow \mathcal{L}$ described in Section 1 , and set about delineating some of its properties, proving (1.1) in the process (see (4.6)). The main message of [3] is that $\theta$ is one-to-one when restricted to those varieties in $Q$ which can be generated by groups of the form $M^{\dagger} B$. We show that when the hypotheses of (1.1) are satisfied every variety in $\mathcal{U}$ has this property. We also give several sufficient conditions on a group $R \in \mathcal{R}$ which ensure that var $R$ is generated by a group of the form $M^{\dagger} B$ (see (4.3)(ii) and (4.5)).

It is convenient to begin with the following lemma: see [1; page 97] for a definition of s-critical generation.

(4.1) LEMMA. (i) An irredundant generating set of basic groups for a Cross variety is an $\mathrm{S}$-critical generating set.

(ii) Let $\left\{X_{1}, X_{2}, \ldots, X_{t}\right\}$ be an s-critical generating set for a Cross variety $\operatorname{var} X$.

(a) In every representation of $X$ as a quotient of a subgroup of a direct product of groups from $\mathrm{QS}\left\{X_{1}, X_{2}, \ldots, X_{t}\right\}$ every one of $X_{1}, X_{2}, \ldots, X_{t}$ occurs.

(b) If $X$ is s-critical then for some $i, X / \Phi(X) \cong X_{i} / \Phi\left(X_{i}\right)$.

Proof. (i) Suppose that $\mathfrak{X}=\left\{X_{1}, X_{2}, \ldots, X_{t}\right\}$ is a set of basic groups irredundantly generating var $\mathcal{X}$. If $\mathcal{X}$ is nots-critical then for some $i$

$$
\operatorname{var} \mathcal{X}=\operatorname{var}\left\{X_{j}: j \neq i\right\} \vee \operatorname{var}(\mathrm{Qs}-1) X_{i} \text {. }
$$

But now the modular law, and the fact that var $X_{i}$ is join irreducible gives either

$$
\operatorname{var} X_{i} \subseteq \operatorname{var}\left\{X_{j}: j \neq i\right\}
$$


or

$$
\operatorname{var} X_{i}=\operatorname{var}(\mathrm{QS}-1) X_{i} \text {. }
$$

The first of these contradicts the irredundance of $\mathscr{X}$ and the second the criticality of $X_{i}$. Therefore, $\mathcal{X}$ is s-critical.

(ii)(a) Since $\operatorname{var} X=\operatorname{QSD}\left\{X_{1}, X_{2}, \ldots, X_{t}\right\}$ (15.73 of [7]) we may write $X=U / V$ where $U$ is a subgroup of a direct product $D_{1} \times D_{2} \times \cdots \times D_{s}$ with each $D_{i} \in \operatorname{Qs}\left\{X_{1}, X_{2}, \ldots, X_{t}\right\}$. Suppose that some $X_{j}$ does not occur in the set $\left\{D_{1}, D_{2}, \ldots, D_{s}\right\}$. Then

$$
X_{j} \in \operatorname{var} X=\operatorname{var}\left\{X_{k}: k \neq j\right\} \vee \operatorname{var}(\mathrm{Qs}-1) X_{j},
$$

contradicting the s-criticality of $\left\{X_{1}, X_{2}, \ldots, X_{t}\right\}$.

(b) Let $P$ be the projective cover of $X$ in $\operatorname{var} X$. By (3.1) of [3] $P$ is s-critical. Let $P_{1}, P_{2}, \ldots, P_{t}$ be the projective covers of $X_{1}, X_{2}, \ldots, X_{t}$ in var $X$. Since $\{P\}$ is the s-critical basis for var $X$ ([2; page 172, paragraph 2]) it follows from Theorem 3.3 of [1] that every $X_{i}$ is a homomorphic image of $P$. Since $P$ is projective, therefore, $P_{i}$ is also a homomorphic image of $P$. But since an s-critical refinement of $\left\{P_{1}, P_{2}, \ldots, P_{t}\right\}$ is also the s-critical basis for var $X([2$; loc. cit.] $P$ is isomorphic to a subgroup of some $P_{i}$. Hence $P \cong P_{i}$. Finally, therefore

$$
X / \Phi(X) \cong P / \Phi(P) \cong P_{i} / \Phi\left(P_{i}\right) \cong X_{i} / \Phi\left(X_{i}\right)
$$

as required.

The notation of the next lemma follows that of Section 1.

(4.2) LeMma. (i) Every critical group in $\mathfrak{A} \mathfrak{B}$ which is of nilpotent length $n+1$ precisely is of the form $M B$ where: $M$ is an abelian normal $p$-subgroup for some prime $p$, complemented by $B ; B \in \mathfrak{B}$ and $B / O_{p}(B)$ has nilpotent length $n$ precisely; $O_{p^{\prime}}(B) \neq 1$ and every non-trivial normal $p^{\prime}$-subgroup of $B$ acts fixed-point-freely on $M$.

(ii) Let $B_{0} \in \mathfrak{B}$ be a finite group and $p$ a prime. Suppose that $O_{p^{\prime}}\left(B_{0}\right) \neq 1$ and that $B_{0} / O_{p}\left(B_{0}\right)$ has nilpotent length $n$ precisely. If $M_{0}$ is a monolithic $Z_{p^{a}} B_{0}-$ module, faithful for $B_{0}$, with $p^{\alpha} \mid r\left(\right.$ so that $\left.M_{0} B_{0} \in \mathfrak{A}, \mathfrak{B}\right)$, then

$$
\operatorname{var} M_{0} B_{0} \in \mathcal{L} \text {. }
$$

Proof. (i) Let $C \in \mathfrak{A} \mathfrak{B}$ be critical and of Fitting height $n+1$ precisely, and let $M$ be the $n$th nilpotent residual of $C$. Now, $M$ is non-trivial, is abelian and, by [5; VI, 7.15], has a complement $B$ in $C$. Since $C$ is monolithic it follows that $M$ is a $p$-group for some prime $p$ and that $C_{B}(M)=1$. Hence, in particular, $\mathfrak{B}(B)=1$. Also, $O_{p^{\prime}}(B) \neq 1$ or $C$ has Fitting height $n$ at most. If $1 \neq N \unlhd B$ and $N \leqslant O_{p^{\prime}}(B)$ then $N$ acts without fixed points on $M$ by Fitting's Lemma. Finally, $B / O_{p}(B)$ has Fitting height $n$ or else $C$ has Fitting height at most $n$. 
(ii) Let $C_{1}, C_{2}, \ldots, C_{s}$ be basic groups generating var $M_{0} B_{0}$ irredundantly. In a minimal representation of $M_{0} B_{0}$ as a quotient of a subgroup of a direct product of groups from QS $\left\{C_{1}, C_{2}, \ldots, C_{s}\right\}$ all $C_{i}$ must occur by (4.1). Now (1.12) of [7] yields that the monolith $\sigma C_{i}$ of $C_{i}$ is similar in $C_{i}$ to the monolith of $M_{0} B_{0}$ in $M_{0} B_{0}$. In particular,

$$
C_{i} / \mathrm{C}_{C_{i}}\left(\sigma C_{i}\right) \cong M_{0} B_{0} / \mathrm{C}_{M_{0} B_{0}}\left(\sigma M_{0} B_{0}\right) \cong B_{0} / O_{p}\left(B_{0}\right),
$$

which has Fitting height $n$. It follows that $C_{i}$ has Fitting height $n+1$ and therefore, $\operatorname{var}\left\{C_{1}, C_{2}, \ldots, C_{2}\right\} \in \mathcal{L}$ as required.

We begin now an analysis of the groups in $R$ with the aim of reducing their number to a canonical set of basic groups still rich enough to generate all varieties in $\mathcal{~}$.

Let $R \in \Re$ : its structure is described in (2.1). Since $A$ has class 2 it follows from the well-known Oates-Powell result (51.37 in [9]) that $A / A^{\prime}$ is either co-monolithic as a $B$-module (that is, has a unique maximal $B$-submodule) or is a sum of at most two (necessarily co-monolithic) proper submodules. It is the second of these possibilities we consider now.

(4.3) LEMMA. Let $R \in \Re$, and, in the notation of (2.1), regard $M=A / A^{\prime}$ as a symplectic $B$-module with scalar product induced by commutation in $A$. Suppose that $M=M_{1}+M_{2}$ for submodules $M_{1}, M_{2}$ and define $\chi: M_{2} \rightarrow M_{1}^{*}$ as in (3.2)(ii)(a). Then

(i) $\chi^{\dagger} B \in \operatorname{var} R$.

If $M_{1}, M_{2}$ are proper submodules of $M$ then

(ii) $\operatorname{var} \chi^{\dagger} B=\operatorname{var} R$

and, in fact,

(iii) for some group $B_{0}$ and $Z_{p^{\alpha}} B_{0}$-module $M_{0}$ for which $M_{0} B_{0}$ is a basic group,

$$
\operatorname{var} R=\operatorname{var} M_{0}^{\dagger} B_{0} \text {. }
$$

ProOF. (i) By (3.7) $M_{1} \chi M_{2}$ is isometric to a submodule of $M Y M Y M$. Let $A_{0}=A Y A Y A$ be a central product realizing $M Y M Y M$. Some subgroup $A_{1}$ of $A_{0}$ therefore realizes $M_{1} \chi M_{2}$. It follows from (3.4) that var $A_{1} B=\operatorname{var} \chi^{\dagger} B$. Hence $\chi^{\dagger} B \in$ var $R$.

(ii) Conversely, (3.6) yields that, for some submodule $U$ of $M_{1} Y M_{1} \chi M_{2} \curlyvee M_{2}$ there is a submodule $K$ contained in $U^{\perp}$ with $U / K$ isometric to $M$. Suppose that $A_{1} / A^{\prime}=M_{1}, A_{2} / A^{\prime}=M_{2}$ so that $A_{1}, A_{2}$ realize $M_{1}, M_{2}$ resectively. There is a subgroup $A_{3}$ of a central product $A_{1} Y \chi^{\dagger} \mathrm{Y} A_{2}$ which realizes $U$. Consequently

$$
A_{3} B \in \operatorname{var}\left\{A_{1} B, A_{2} B, \chi^{\dagger} B\right\} \text {. }
$$


If $\mu: A_{3} \rightarrow U$ is the natural $B$-homomorphism write $K_{0}=K \mu^{-1}$. Then $K_{0}$ is central in $A_{3}$. However, $O_{p^{\prime}}(B)$ has no fixed points in $M$ and therefore none in $U$. Hence $X_{3}=\operatorname{ker} \mu$ is precisely the set of fixed points of $O_{p^{\prime}}(B)$ in $K_{0}$ and Fitting's Lemma therefore yields

$$
K_{0}=X_{3} \times V
$$

where $V$ admits $B$. But now $A_{3} / V$ realizes $M$ since $U / K$ is isometric to $M$. Hence by (3.4) $\operatorname{var}\left(A_{3} / V\right) B=\operatorname{var} R$ and, in consequence, (4.4) yields

$$
R \in \operatorname{var}\left\{A_{1} B, A_{2} B, \chi^{\dagger} B\right\} .
$$

However, $A_{1} B, A_{2} B$ and $\chi^{\dagger} B$ belong to var $R$. In particular, the modular law gives

$$
\operatorname{var} R=\operatorname{var} \chi^{\dagger} B \vee\left(\operatorname{var} R \wedge \operatorname{var}\left\{A_{1} B, A_{2} B\right\}\right) .
$$

Since $R$ is basic the only possibility is $\operatorname{var} R=\operatorname{var} \chi^{\dagger} B$, as required.

(iii) We now know that $\operatorname{var} R$ is generated by a split extension $C B^{0}$ with the following properties: $C$ is a $B^{0}$-group, a $p$-group of class 2 with abelian $B^{0}$-subgroups $C_{1}, C_{2}$ which together generate $C ; C_{1} \cap C_{2}=1$ and $C^{\prime}=\left[C_{1}, C_{2}\right]$ is cyclic and central in $C B^{0} ; O_{p^{\prime}}(B)$ is non-trivial and has no fixed points in $C_{1}$ or in $C_{2}$; and $B^{0} / O_{p}\left(B^{0}\right)$ has Fitting height $n$ precisely. Among all generating groups for $\operatorname{var} R$ with these properties suppose that $C B^{0}$ is the smallest.

First we show that the maps $\chi_{1}: C_{2} \rightarrow C_{1}^{*}$ and $\chi_{2}: C_{1} \rightarrow C_{2}^{*}$ ensured by (3.2) must both be one-to-one: since ker $\chi_{i} \unlhd C B^{0}$ and ker $\chi_{i} \cap C^{\prime}=1$ it follows that

$$
\operatorname{var} R=\operatorname{var} C B^{0} / \operatorname{ker} \chi_{i} \vee \operatorname{var} C B^{0} / C^{\prime} \text {; }
$$

whence

$$
\operatorname{var} R=\operatorname{var} C B^{0} / \operatorname{ker} \chi_{i}
$$

since var $R$ is join irreducible; whence ker $\chi_{i}=1$ by the minimality of $C B^{0}$.

By (2.2), therefore, $C \cong C_{1}^{\dagger}$ as $B^{0}$-group. The minimality ensures that $C_{1}$ is faithful for $B^{0}$ and also that $C_{1}$ is monolithic and co-monolithic as $B^{0}$-module (using (2.3) in this case).

Finally, suppose that $\Gamma=C_{1} B^{0}$ is not basic. By (4.2) var $\Gamma$ is generated by basic groups $\Gamma_{i}=M_{i} B_{i}(0 \leqslant i \leqslant s)$. Hence we can deduce from (2.3) that

$$
\operatorname{var} R=\operatorname{var} C_{1}^{\dagger} B^{0}=\operatorname{var}\left\{M_{i}^{\dagger} B_{i}: 0 \leqslant i \leqslant s\right\} .
$$

Since $\operatorname{var} R$ is join irreducible $\operatorname{var} R=\operatorname{var} M_{0}^{\dagger} B_{0}$ say, as required.

The next result gives a very different condition ensuring a result like (4.3)(ii). 
(4.5) LeMma. Let $R \in \Re$. Regard $M=A / A^{\prime}$ as a non-degenerate symplectic $B$-module in the usual way. If End $M$ contains a symplectic endomorphism $e$ such that $1-e$ is invertible then

$$
\operatorname{var} R=\operatorname{var} M^{\dagger} B
$$

Proof. By (3.7) $M \iota M^{*}$ and $M Y M$ are isometric. Since $M^{\dagger}$ realizes the first and a central product $A Y A$ the second we have the desired result from (3.4).

Let $\Re^{\dagger}$ be the set of those basic groups in $R$ which satisfy the hypotheses of (4.3) or (4.5). That is to say $R \in R^{\dagger}$ if and only if either $A / A^{\prime}$ is not co-monolithic or $\operatorname{End}_{B} A / A^{\prime}$ contain a symplectic endomorphism $e$ such that $1-e$ is invertible. Let $\mathcal{Q}^{\dagger}$ be the subset of $\mathcal{Q}$ of those varieties generated by groups from $\mathfrak{R}^{\dagger}$.

The main result of this section can now be stated.

(4.6) ThEOREM. (i) There is a function $\theta: \mathcal{Q} \rightarrow \mathcal{L}$ defined by

$$
\mathfrak{X} \theta=\operatorname{var}\left\{H / \zeta_{1}(H): H \in \mathfrak{X}\right\}, \quad \mathfrak{X} \in \mathscr{Q} .
$$

(ii) The restriction of $\theta$ to $\mathscr{Q}^{\dagger}$ is one-to-one.

(iii) $\mathcal{U}^{\dagger}=\mathscr{Q}$ if either $\mathfrak{B}$ is nilpotent, $r$ is odd or the exponent of $\mathfrak{B}$ is odd.

Proof. (i) We must show that $\mathfrak{X} \theta \in \mathcal{L}$ whenever $\mathfrak{X} \in \mathcal{U}$ and for this it suffices that $\operatorname{var} R / \zeta_{1}(R) \in \mathcal{L}$ whenever $R \in \mathcal{R}$. Now either $A / A^{\prime}$ is co-monolithic or it is not. In the first case it is also monolithic, being self-contragredient by (3.2)(ii)(b), and hence $\operatorname{var} R / \zeta_{1}(R) \in \mathcal{L}$ by (4.2)(ii). In the second case $\operatorname{var} R=\operatorname{var} M_{0}^{\dagger} B_{0}$ where $M_{0} B_{0}$ is basic, by (4.3). Hence

$$
\operatorname{var} R / \zeta_{1}(R)=\operatorname{var}\left\{M_{0} B_{0}, M_{0}^{*} B_{0}\right\} \in \mathcal{L}
$$

since $M_{0} B_{0}$ and $M_{0}^{*} B_{0}$ both satisfy the hypotheses of (4.3)(ii).

(ii) Now suppose that $\mathfrak{X}_{1}, \mathfrak{X}_{2} \in \mathcal{Q}^{\dagger}$ with $\mathfrak{X}_{1} \theta \leqslant \mathfrak{X}_{2} \theta$. By (4.3) and (4.5) we may write

$$
\mathfrak{X}_{i}=\operatorname{var}\left\{M_{i j}^{\dagger} B_{i j}: j \in I(i)\right\}, \quad i=1,2,
$$

where all $M_{i j} B_{i j}$ are monolithic groups. Then, since $\mathfrak{X}_{1} \theta \leqslant \mathfrak{X}_{2} \theta$,

$$
M_{i j} B_{i j} \in \operatorname{var}\left\{M_{2 k} B_{2 k}, M_{2 k}^{*} B_{2 k}: k \in I(2)\right\}, \quad j \in I(1) .
$$

By (2.3), therefore,

$$
M_{i j}^{\dagger} B_{i j}^{\dagger} \in \operatorname{var}\left\{M_{2 k}^{\dagger} B_{2 k}: k \in I(2)\right\}, \quad j \in I(1),
$$

and so $\mathfrak{X}_{1} \leqslant \mathfrak{X}_{2}$. This is more than enough to prove $\theta$ is one-to-one. 
(iii) Suppose that $\mathfrak{B}$ is nilpotent. It follows that if $R \in \mathscr{R}, \zeta_{1}(B) \cap O_{p^{\prime}}(B) \neq 1$, so we may choose a non-trivial element $k \in \zeta_{1}(B) \cap O_{p^{\prime}}(B)$. Since $\langle k\rangle \unlhd B$, $\left[A / A^{\prime}, k\right]=A / A^{\prime}$ by $(2.1)(\mathrm{d})$. It follows that, if we denote the linear map $A / A^{\prime} \rightarrow A / A^{\prime}$ induced by conjugation with $k$ also by $k$, then $1-k$ is onto and therefore invertible. Since $k \in \zeta_{1}(B), 1-k \in \mathrm{End}_{B} A / A^{\prime}$; and, since $k$ centralizes $A^{\prime}$, it induces an isometry in $A / A^{\prime}$ by conjugation. By (4.5), therefore, $R \in \mathbb{R}^{\dagger}$. Hence $\mathcal{U}^{\dagger}=\mathcal{Q}$.

Next, suppose that $r$ is odd. The map $e: a+A^{\prime} \rightarrow-a+A^{\prime}$ is then an isometry for which $1-e$ is invertible. Again by (4.5), $\mathcal{Q}^{\dagger}=\mathscr{Q}$.

To conclude the proof of (4.6) I prove the following results.

(4.7) Theorem. (See Isaacs [5; p. 276].) Let $P$ be $Z_{p^{\alpha}}$ or a field and $M a$ $P B$-module of finite P-rank. Suppose that $M$ admits an invariant, non-degenerate scalar product $\langle\rangle:, M \times M \rightarrow P$. Then $E=$ End $M$ admits an algebra anti-automorphism $e \mapsto e^{*}$ satisfying $e^{* *}=e$ and $\left\langle m e, m^{\prime}\right\rangle=\left\langle m, m^{\prime} e^{*}\right\rangle\left(m, m^{\prime} \in M\right)$.

Proof. Since the scalar product is non-degenerate the homomorphism $\chi$ : $M \rightarrow M^{*}$ ensured by (3.2) is one-to-one and therefore onto. For each $e \in E$ and $m \in M$ the function $M \rightarrow P$ defined by $m^{\prime} \mapsto\left\langle m^{\prime} e, m\right\rangle$ is linear and hence belongs to $M^{*}$. Therefore for some $m_{0} \in M, \chi_{m_{0}}\left(m^{\prime}\right)=\left\langle m^{\prime} e, m\right\rangle$ and $m_{0}$ is uniquely determined by $m$ and $e$. However, the map $M \rightarrow M$ defined by $m \mapsto m_{0}$ is in $E$; denote it by $e^{*}$. It is then easy to check that $e \mapsto e^{*}$ satisfies the properties claimed.

We call $e^{*}$ the adjoint of $e$.

(4.8) ThEOREM. If $K$ has odd order and if $M$ is a faithful irreducible $Z_{2} K$-module admitting a non-degenerate invariant scalar product then End $M$ contains a nonidentity isometry.

Proof. Let $a \in K$ be an arbitrary element different from 1. Then $a$ and $a^{-1}$ are not conjugate in $K$. For if $a^{k}=a^{-1}$ for some $k \in K$ we should have that $k^{2}$ centralizes $a$, then that $k$ centralizes $a$ since $k$ has odd order, whence $a=a^{-1}$ or $a^{2}=1$, and finally $a=1$. It follows that the conjugacy classes [a] of $A$, other than that consisting of the identity element alone, may be paired off

$$
[a] \leftrightarrow\left[a^{-1}\right] .
$$

Consequently, representatives of the conjugacy classes other than [1] may be chosen in the form $a_{1}, a_{1}^{-1}, a_{2}, a_{2}^{-1}, \ldots, a_{r}, a_{r}^{-1}$.

Let us denote by $\sigma(a)$ the sum of the linear transformations induced in $M$ by the elements of the conjugacy class $[a]$. Note that $\sigma(a) \in$ End $M$ for all $a \in K$. 
Also, $\sigma(a)^{*}=\sigma\left(a^{-1}\right)$ since, for all $m, m^{\prime} \in M$,

$$
\begin{aligned}
\left\langle m \sigma(a), m^{\prime}\right\rangle & =\left\langle m \sum_{t \in T} a^{t}, m^{\prime}\right\rangle=\sum_{t \in T}\left\langle m a^{t}, m^{\prime}\right\rangle=\sum_{t \in T}\left\langle m, m^{\prime} a^{-t}\right\rangle \\
& =\left\langle m, m^{\prime} \sum_{t \in T} a^{-t}\right\rangle=\left\langle m, m^{\prime} \sigma\left(a^{-1}\right)\right\rangle .
\end{aligned}
$$

Here $T$ is a complete set of coset representatives for $\mathbf{C}_{K}(a)$ in $K$.

We show that for some $a \in K, \sigma(a) \neq \sigma\left(a^{-1}\right)$. For it, to the contrary, $\sigma(a)=$ $\sigma\left(a^{-1}\right)$ for all $a \in K$ then

$$
e=\sum_{x \in K} x=1+\sum_{i=1}^{r}\left[\sigma\left(a_{i}\right)+\sigma\left(a_{i}^{-1}\right)\right]=1,
$$

since we are working modulo 2 . However, for all $y \in K$,

$$
y=y e=e=1 \text {, }
$$

a contradiction to the non-triviality of $K$.

We therefore have an element $f \in$ End $M$ for which $f^{*} \neq f$. Hence since End $M$ is a field, so that the adjoint operator is an automorphism of order 2 on End $M$, the multiplicative group splits

$$
\text { End } M^{\times}=F \times N
$$

where $f^{*}=f$ for all $f \in F, \varepsilon^{*}=\varepsilon^{-1}$ for all $\varepsilon \in N$, and $N \neq 1$. If $1 \neq \varepsilon \in N$ therefore

$$
\left\langle m \varepsilon, m^{\prime} \varepsilon\right\rangle=\left\langle m, m^{\prime} \varepsilon \varepsilon^{*}\right\rangle=\left\langle m, m^{\prime}\right\rangle
$$

for all $m, m^{\prime} \in M$ and $\varepsilon$ is an isometry, as required.

(4.9) COROLLARY. If $K$ is finite and of odd order and if $M$ is a non-degenerate symplectic $Z_{2^{\alpha}} K$-module such that $M / 2 M$ is irreducible then End $M$ contains a non-identity isometry.

Proof. The proof will be by induction on $\alpha$, the case $\alpha=1$ being (4.8). So suppose $\alpha>1$.

The given scalar product $M \times M \rightarrow P\left(=Z_{2^{a}}\right)$ induces a non-degenerate scalar product on $M / 2^{\beta} M(1 \leqslant \beta \leqslant \alpha-1)$; in fact $\langle\rangle:, M / 2^{\beta} M \times M / 2^{\beta} M \rightarrow P / 2^{\beta} P$ :

$$
\left\langle m_{1}+2^{\beta} M, m_{2}+2^{\beta} M\right\rangle=\left\langle m_{1}, m_{2}\right\rangle+2^{\beta} P .
$$

If $\theta$ is an isometric endomorphism of $M / 2 M$, different from 1 , it may be lifted using the Schur-Zassenhaus Theorem to an endomorphism of $M$. By induction we may assume that on $M / 2^{\alpha-1} M, \theta$ is an isometry. Hence

$$
\left\langle m_{1} \theta, m_{2} \theta\right\rangle-\left\langle m_{1}, m_{2}\right\rangle \in 2^{\alpha-1} P
$$


for all $m_{1}, m_{2} \in M$. We suppose that $\theta$ is in fact not an isometry on $M$. Then, it is easy to check that the function [, ]: $M / 2 M \times M / 2 M \rightarrow P / 2 P$ defined by

$$
\left[m_{1}+2 M, m_{2}+2 M\right]=\frac{1}{2^{\alpha-1}}\left(\left\langle m_{1} \theta, m_{2} \theta\right\rangle-\left\langle m_{1}, m_{2}\right\rangle\right)+2 P
$$

is a non-degenerate scalar product. By (3.8)(i) [, ] must be isometric to $\langle$,$\rangle on$ $M / 2 M$ so that for some $\varphi \in$ End $M / 2 M$

$$
\begin{aligned}
{\left[m_{1}+2 M, m_{2}+2 M\right] } & =\left\langle\left(m_{1}+2 M\right) \varphi,\left(m_{2}+2 M\right) \varphi\right\rangle \\
& =\left\langle m_{1} \varphi+2 M, m_{2} \varphi+2 M\right\rangle,
\end{aligned}
$$

lifting $\varphi$ to $M$, so

$$
\frac{1}{2^{\alpha-1}}\left(\left\langle m_{1} \theta, m_{2} \theta\right\rangle-\left\langle m_{1}, m_{2}\right\rangle\right)+2 P=\left\langle m_{1} \varphi, m_{2} \varphi\right\rangle+2 P
$$

or

$$
\left\langle m_{1} \theta, m_{2} \theta\right\rangle=\left\langle m_{1}, m_{2}\right\rangle+2^{\alpha-1}\left\langle m_{1} \varphi, m_{2} \varphi\right\rangle
$$

Therefore,

$$
\begin{aligned}
\left\langle m_{1} \theta^{2}, m_{2} \theta^{2}\right\rangle= & \left\langle m_{1} \theta, m_{2} \theta\right\rangle+2^{\alpha-1}\left\langle m_{1} \theta \varphi, m_{2} \theta \varphi\right\rangle \\
= & \left(\left\langle m_{1}, m_{2}\right\rangle+2^{\alpha-1}\left\langle m_{1} \varphi, m_{2} \varphi\right\rangle\right)+2^{\alpha-1}\left\langle m_{1} \varphi \theta, m_{2} \varphi \theta\right\rangle \\
= & \left(\left\langle m_{1}, m_{2}\right\rangle+2^{\alpha-1}\left\langle m_{1} \varphi, m_{2} \varphi\right\rangle\right) \\
& +2^{\alpha-1}\left(\left\langle m_{1} \varphi, m_{2} \varphi\right\rangle+2^{\alpha-1}\left\langle m_{1} \varphi^{2}, m_{2} \varphi^{2}\right\rangle\right) \\
= & \left\langle m_{1}, m_{2}\right\rangle .
\end{aligned}
$$

But $\theta$ has odd multiplicative order so $\theta$ is also an isometry. This completes the induction and the proof of (4.9).

The proof of (4.6) is now completed using (4.5) in the usual way.

At the risk of labouring the point I finish by pointing out again that when generating groups of the type $M^{\dagger} B$ can be found there is no trouble. The difficulties arise when $r$ is a power of 2 , when $A / A^{\prime}$ is co-monolithic and when $A / A^{\prime}$ has no symplectic endomorphisms satisfying (4.5) (in particular $B$ has trivial centre). The smallest instance of such a group $R$ is $G L(2,3): A \cong Q_{8}$, the quaternion group of order 8 , and $B \cong S_{3}$, the symmetric group on three letters, and in this case $\operatorname{var} M^{\dagger} B \neq \operatorname{var} R$. For, it is easy to check that the Sylow 2-subgroups of $R$ here have exponent 8 whilst those of $M^{\dagger} B$ have exponent 4.

I propose to investigate such cases in a sequel which, in contrast to the present paper, will be concerned with the questions "How badly can $\theta$ fail to be one-to-one?", and to what extent are the conditions derived here, which ensure $\operatorname{var} R=\operatorname{var} M^{\dagger} B$, also necessary? 


\section{References}

[1] Roger M. Bryant, 'On s-critical groups', Quart. J. Math. Oxford Ser. (2) 22 (1971), 91-101.

[2] Roger M. Bryant, 'Finte splitting groups in varieties of groups', Quart. J. Math. Oxford Ser. (2) 22 (1971), 169-172.

[3] R. A. Bryce, 'Centre-by-abelian-by-nilpotent varieties of groups', J. London Math. Soc. (2) 12 (1976), 337-350.

[4] Robert L. Greiss, Jr., 'Automorphisms of extra-special groups and non-vanishing degree 2 cohomology', Pacific J. Math. 48 (1973), 403-422.

[5] B. Huppert, Endliche Gruppen I (Springer-Verlag, Berlin-Heidelberg-New York, 1967).

[6] I. Martin Isaacs, 'Primitive characters, normal subgroups, and M-groups', Math. Z. 177 (1981), 267-284.

[7] L. G. Kovács and M. F. Newman, 'On critical groups', J. Austral. Math. Soc. 6 (1966), 237-250.

[8] B. H. Neumann, 'Groups with finite classes of conjugate elements', Proc. London Math. Soc. (3) 1 (1951), 178-187.

[9] Hanna Neumann, Varieties of groups (Springer-Verlag, Berlin-Heidelberg-New York, 1967).

Department of Mathematics

Faculty of Science

Australian National University

P. O. Box 4

Canberra A.C.T. 2601

Australia 